\title{
Numerical methods for characterization of synchrotron radiation based on the Wigner function method
}

\author{
Takashi Tanaka* \\ RIKEN SPring-8 Center, Koto 1-1-1, Sayo, Hyogo 679-5148, Japan
}

(Received 16 April 2014; published 27 June 2014)

\begin{abstract}
Numerical characterization of synchrotron radiation based on the Wigner function method is explored in order to accurately evaluate the light source performance. A number of numerical methods to compute the Wigner functions for typical synchrotron radiation sources such as bending magnets, undulators and wigglers, are presented, which significantly improve the computation efficiency and reduce the total computation time. As a practical example of the numerical characterization, optimization of betatron functions to maximize the brilliance of undulator radiation is discussed.
\end{abstract}

DOI: 10.1103/PhysRevSTAB.17.060702

PACS numbers: 41.60.Ap

\section{INTRODUCTION}

Recent progress of accelerator theories and technologies has made it possible to generate an electron beam whose emittance is comparable to that of diffraction limited light even in the angstrom x-ray region, both in the storagering and energy-recovery-linac schemes. These novel accelerator-based synchrotron radiation (SR) facilities are expected to improve the spatial coherence of SR to an unprecedented level and will be complementary to the $\mathrm{x}$-ray free electron lasers as pulsed $\mathrm{x}$-ray sources. It should be noted, however, that it is necessary to accurately evaluate the characteristics of radiation in order to take full advantage of the high source quality to be realized in such advanced SR facilities.

In the framework of geometrical optics, light can be described as a point particle (photon) having definite coordinates and its motion can be fully characterized by four coordinate variables under a paraxial approximation. To be more specific, the photons move in the 4-dimensional (4D) phase space spanned by $\boldsymbol{r}=(x, y)$ and $\boldsymbol{\theta}=\left(\theta_{x}, \theta_{y}\right)$, namely, positions and angles in the horizontal and vertical directions. Although the above description of photon motion is convenient for a lot of applications, it is not allowed in wave optics in which diffraction plays an important role for propagation of light and the two coordinates $x$ and $\theta_{x}$ cannot be defined simultaneously. Even so, we define the photon density $d(\boldsymbol{r}, \boldsymbol{\theta})$ in the $4 \mathrm{D}$ phase space (hereinafter, phase-space density) for a number of practical reasons as follows.

First, its distribution function is required to carry out the ray-trace simulation based on the geometrical optics, which

\footnotetext{
"ztanaka@ spring8.or.jp

Published by the American Physical Society under the terms of the Creative Commons Attribution 3.0 License. Further distribution of this work must maintain attribution to the author(s) and the published article's title, journal citation, and DOI.
}

is widely used to design the optical components in the SR beam line. Second, its maximum value, which is usually referred to as brilliance or brightness, gives us good information on how many coherent photons are available and can be brought to the sample.

A number of computer codes have already been developed for numerical characterization of SR, in which the photon distribution is given either as a far-field pattern or as a near-field pattern. The former refers to the photon flux per unit solid angle, $f_{a}(\boldsymbol{\theta})$, which is obtained by integrating $d(\boldsymbol{r}, \boldsymbol{\theta})$ over $\boldsymbol{r}$. The latter refers to the photon flux per unit area, $f_{s}(\boldsymbol{r})$, which is obtained by integrating $d(\boldsymbol{r}, \boldsymbol{\theta})$ over $\boldsymbol{\theta}$. Note that analytical formulas of the two functions $f_{a}$ and $f_{s}$ are explicitly derived from classical electrodynamics and numerical computations of them are rather straightforward. This is not the case for the function $d(\boldsymbol{r}, \boldsymbol{\theta})$, whose mathematical expression cannot be derived only within the framework of classical electrodynamics. As a result, a Gaussian approximation is usually made, in which $d(\boldsymbol{r}, \boldsymbol{\theta})$ is approximated by a 4D Gaussian function whose standard deviations are defined by the functions $f_{a}$ and $f_{s}$. It should be noted, however, that the Gaussian approximation in the characterization of SR is not actually proven to be validated in all cases, i.e., the accuracy and reliability of this method are not clear.

In order to overcome the above difficulty, characterization of SR based on the cross-spectral density has been performed by several authors, especially focused on the transverse coherence properties of undulator radiation (UR). In [1], a rigorous expression for the cross-spectral density, with the electron beam distribution taken into account, has been derived together with its asymptotic form, and the coherence properties have been investigated in a comprehensive way. In [2], a phenomenological approach, which is based on an assumption that radiation is generated by a Gaussian Schell-model source, has been successfully introduced. 
Another way of accurately characterizing SR is based on the so-called Wigner function [3], which is directly related to $d(\boldsymbol{r}, \boldsymbol{\theta})$ and has been introduced by several authors [4-9]. In these papers, analytical and numerical methods have been discussed to calculate the phase-space density of UR emitted from a single electron, which makes clear the difference between the exact and Gauss-approximated profiles of $d(\boldsymbol{r}, \boldsymbol{\theta})$. It should be noted, however, that the effects due to the finite emittance and energy spread of the electron beam have not been taken into account except for a few examples. The reason for this will most probably be the fact that numerical computation of $d(\boldsymbol{r}, \boldsymbol{\theta})$ under such a "practical" condition results in an eightfold integration and thus requires a huge amount of computation time. Recently, analytical methods to reduce the numerical cost have been presented in [10], which takes advantage of the fact that the dimension of the Wigner function can be reduced from 4 to 3 under the rotational symmetry. This method has been applied to the round electron beam which will be available in an energy-recovery linac or an ultimated storage ring with full coupling. Note, however, that this technique cannot be applied to more common SR sources in which the radial symmetry is not available.

In this paper, numerical methods to compute the phasespace density of SR are explored in more detail for the purpose of reducing the total number of numerical operations and completing the computation within a reasonable time even under the practical conditions. One numerical example is also discussed in order to show the potential and practical applications of the numerical characterization of SR based on the Wigner function method. Note that the bending magnet radiation (BMR) and wiggler radiation (WR), in addition to UR, are also within the scope of this paper, whose Wigner functions have not been seriously considered in the previous papers.

\section{GENERAL FORMULATION OF THE PHASE-SPACE PHOTON DENSITY}

Let us first describe the general method to formulate the phase-space photon density based on the Wigner function method. In the following discussions, let $z$ be the main axis of the electron beam and thus the optical axis of SR, $x$ and $y$ be the horizontal and vertical axes perpendicular to $z$, and the coordinate origin be the center of the SR source.

\section{A. Wigner function of SR emitted by a single electron}

We start with the well-known formula that denotes the frequency-domain electric field of SR emitted from a single electron at the frequency of $\omega$, which is given as [11]

$$
\boldsymbol{E}_{\omega}(\boldsymbol{r})=\frac{i e \omega}{4 \pi \varepsilon_{0} c} \int \frac{1}{R(z)}\left[\boldsymbol{\beta}(z)-\left(1+\frac{i c}{\omega R(z)}\right) \boldsymbol{n}(z)\right] \mathrm{e}^{i \omega \tau(z)} d z,
$$

with

$$
\boldsymbol{R}(z)=\boldsymbol{r}-\boldsymbol{r}_{e}(z), \quad \boldsymbol{n}(z)=\boldsymbol{R}(z) / R(z),
$$

and

$$
\tau(z)=\frac{1}{2 c}\left\{\frac{z}{\gamma^{2}}+\int^{z} \boldsymbol{\beta}_{\perp}^{2}\left(z^{\prime}\right) d z^{\prime}+\frac{\left[\boldsymbol{\rho}-\boldsymbol{\rho}_{e}(z)\right]^{2}}{Z-z}\right\},
$$

where $e$ denotes the electron charge, $c$ the speed of light, $\varepsilon_{0}$ the vacuum permittivity, $\boldsymbol{r}=(X, Y, Z)$ means the vector directing from the origin to the observation point, and $\gamma$ is the Lorentz factor of the electron moving along a trajectory specified by $\boldsymbol{r}_{e}(z)$ with the relative velocity of $\boldsymbol{\beta}(z)$. The $2 \mathrm{D}$ vectors $\boldsymbol{\rho}, \boldsymbol{\rho}_{e}$, and $\boldsymbol{\beta}_{\perp}$ denote the transverse components of the $3 \mathrm{D}$ vectors $\boldsymbol{r}, \boldsymbol{r}_{e}$ and $\boldsymbol{\beta}$, respectively. Note that the integration range depends on the type of the SR source.

The photon density in the $4 \mathrm{D}$ phase space at the longitudinal position $z=Z$ is given by the Wigner function defined as

$$
W(\boldsymbol{\rho}, \boldsymbol{\theta}, Z) \propto \int E_{\omega}^{*}\left(\boldsymbol{\rho}+\frac{\boldsymbol{\rho}^{\prime}}{2}, Z\right) E_{\omega}\left(\boldsymbol{\rho}-\frac{\boldsymbol{\rho}^{\prime}}{2}, Z\right) \mathrm{e}^{i k \boldsymbol{\theta} \cdot \boldsymbol{\rho}^{\prime}} d \boldsymbol{\rho}^{\prime},
$$

where $k=\omega / c$ denotes the wave number of radiation, $\boldsymbol{\rho}=$ $(X, Y)$ the transverse position already defined, $\boldsymbol{\theta}=\left(\theta_{x}, \theta_{y}\right)$ the angle with respect to the optical axis, and $E_{\omega}$ refers to either of the two field components corresponding to horizontal and vertical polarization states. The coefficient of the Wigner function should be determined so that its integration over $\boldsymbol{\rho}$ and $\boldsymbol{\theta}$ gives the total photon flux of the relevant polarization state. For details of the properties of Wigner function, refer to [5] and [9].

Now we find from Eqs. (1) and (2) that the Wigner function is given as a threefold integral. In order to take into account the effects due to the finite emittance and energy spread of the electron beam, we need to convolute the Wigner function $W$ with the 5D distribution function, which means that we need to carry out an eightfold integral to compute the phase-space density under a practical condition. Also note that the phase-space density at the source point $z=0$ is usually desired for characterization of radiation, which requires another numerical operation. To be more specific, the complex amplitude $E_{\omega}(\rho, Z)$ is computed at a certain longitudinal position $z=Z$ at least behind the exit of the SR source, and then is back propagated to the source point $z=0$ by means of the Fresnel-Kirchhoff's diffraction integral [12].

The complicated numerical process explained above to compute the Wigner function at the source point is significantly simplified by introducing an angular representation of the complex amplitude, which has been originally pointed out by Kim [6] and is repeated here in more detail. 
Let us first introduce the spatial Fourier transform of $E_{\omega}(\boldsymbol{\rho}, Z)$ defined as

$$
\mathcal{E}_{\omega}(\boldsymbol{\theta}, Z)=\frac{1}{\lambda^{2}} \int E_{\omega}(\boldsymbol{\rho}, Z) \mathrm{e}^{-i k \theta \cdot \boldsymbol{\rho}} d \boldsymbol{\rho},
$$

where $\lambda=2 \pi / k$ denotes the wavelength of radiation. Then it is easy to show that the Wigner function defined by Eq. (2) can be redefined using $\mathcal{E}_{\omega}$ as follows

$$
W(\boldsymbol{\rho}, \boldsymbol{\theta}, Z) \propto \int \mathcal{E}_{\omega}^{*}\left(\boldsymbol{\theta}+\frac{\boldsymbol{\theta}^{\prime}}{2}, Z\right) \mathcal{E}_{\omega}\left(\boldsymbol{\theta}-\frac{\boldsymbol{\theta}^{\prime}}{2}, Z\right) \mathrm{e}^{-i k \boldsymbol{\theta}^{\prime} \cdot \boldsymbol{\rho}} d \boldsymbol{\theta}^{\prime}
$$

Although both Eqs. (2) and (4) are available to compute the Wigner function, the latter is much more preferable especially for the computation at the source point as explained in what follows.

The inverse transform of $\mathcal{E}_{\omega}$ gives

$$
\begin{aligned}
E_{\omega}(\boldsymbol{\rho}, Z) & =\int \mathcal{E}_{\omega}(\boldsymbol{\theta}, Z) \mathrm{e}^{i k \boldsymbol{\theta} \cdot \boldsymbol{\rho}} d \boldsymbol{\theta} \\
& =\mathrm{e}^{i k \boldsymbol{\rho}^{2} / 2 Z} \int \mathcal{E}_{\omega}(\boldsymbol{\theta}, 0) \mathrm{e}^{-i k Z(\boldsymbol{\theta}-\boldsymbol{\rho} / Z)^{2} / 2} d \boldsymbol{\theta},
\end{aligned}
$$

where we have applied the diffraction theory given as

$$
\mathcal{E}_{\omega}(\boldsymbol{\theta}, Z)=\mathcal{E}_{\omega}(\boldsymbol{\theta}, 0) \mathrm{e}^{-i k Z \boldsymbol{\theta}^{2} / 2},
$$

which is valid under the paraxial approximation [13]. Note that the fast oscillating factor $\mathrm{e}^{i k Z}$ has been omitted in the above equations because it is not important in the following discussions.

Using the relation

$$
\lim _{Z \rightarrow \infty} \mathrm{e}^{-i Z t^{2}}=\mathrm{e}^{-i \pi / 4} \sqrt{\frac{\pi}{Z}} \delta(t),
$$

Eq. (5) can be rewritten as

$$
\mathcal{E}_{\omega}(\boldsymbol{\theta}, 0)=\frac{i}{\lambda} \lim _{Z \rightarrow \infty} Z E_{\omega}(\boldsymbol{\rho}=\boldsymbol{\theta Z}, Z) \mathrm{e}^{-i k Z \boldsymbol{\theta}^{2} / 2},
$$

which means that the angular representation of the complex amplitude is given by the field pattern observed at the position infinitely far from the source point. After substituting $\boldsymbol{\rho}=\boldsymbol{\theta} Z$ into Eq. (1) and taking the limit $Z \rightarrow \infty$, we have the following relations

$$
\begin{aligned}
R(z) & \rightarrow Z, \quad \boldsymbol{n}(z) \rightarrow \boldsymbol{\theta}, \\
\tau(z) & \rightarrow \frac{1}{2 \gamma^{2} c} \int^{z}\left\{1+\gamma^{2}\left[\boldsymbol{\beta}_{\perp}\left(z^{\prime}\right)-\boldsymbol{\theta}\right]^{2}\right\} d z^{\prime}+\frac{k \boldsymbol{\theta}^{2} Z}{2} .
\end{aligned}
$$

As a result, Eq. (6) is simplified to

$$
\mathcal{E}_{\omega}(\boldsymbol{\theta}, 0)=-\frac{e}{2 \varepsilon_{0} c \lambda^{2}} \int\left[\beta_{x, y}(z)-\theta_{x, y}\right] \mathrm{e}^{i \omega \tau(z)} d z,
$$

with

$$
\tau(z)=\frac{1}{2 \gamma^{2} c} \int^{z}\left\{1+\gamma^{2}\left[\boldsymbol{\beta}_{\perp}\left(z^{\prime}\right)-\boldsymbol{\theta}\right]^{2}\right\} d z^{\prime},
$$

where either of the two subscripts $x$ and $y$ should be chosen according to the relevant polarization state. The above equation to compute $\mathcal{E}_{\omega}(\boldsymbol{\theta}, 0)$ is much simpler than Eq. (1) to compute $E_{\omega}$. Furthermore, it is more convenient in the point that one can take advantage of numerical techniques that are familiar to the formulation of SR, such as an expansion by Bessel functions, and an application of the periodic condition of undulator magnetic fields, to be discussed later. Also note that no special numerical operation is required to compute $\mathcal{E}_{\omega}$ at the source point. This is not the case for $E_{\omega}$, which cannot be directly computed at the source point.

\section{B. Normalization}

Now let us introduce several normalizations to make easier the further discussions. In the case of typical SR sources as discussed later, it is convenient to normalize the coordinate variables $\boldsymbol{\rho}$ and $\boldsymbol{\theta}$ as follows

$$
\hat{\boldsymbol{\rho}}=(U, V) \equiv \boldsymbol{\rho} / \chi, \quad \hat{\boldsymbol{\theta}}=(u, v) \equiv \boldsymbol{\theta} / \chi^{\prime},
$$

where $\chi$ and $\chi^{\prime}$ are the angular and positional normalization factors to be defined according to the type of the SR source, and $U(u)$ and $V(v)$ are the normalized positions (angles) in the horizontal and vertical directions, respectively. Using these normalized coordinates, we define the normalized Wigner function as

$$
\hat{W}(\hat{\boldsymbol{\rho}}, \hat{\boldsymbol{\theta}})=\int \mathcal{W}\left(\hat{\boldsymbol{\theta}}, \hat{\boldsymbol{\theta}}^{\prime}\right) \mathrm{e}^{-i \hat{\boldsymbol{\theta}}^{\prime} \cdot \hat{\boldsymbol{\rho}}} d \hat{\boldsymbol{\theta}}^{\prime},
$$

where $\mathcal{W}$ is a bilinear function given by

$$
\mathcal{W}\left(\hat{\boldsymbol{\theta}}, \hat{\boldsymbol{\theta}}^{\prime}\right)=\hat{\mathcal{E}}^{*}\left(\hat{\boldsymbol{\theta}}+\frac{\hat{\boldsymbol{\theta}}^{\prime}}{2}\right) \hat{\mathcal{E}}\left(\hat{\boldsymbol{\theta}}-\frac{\hat{\boldsymbol{\theta}}^{\prime}}{2}\right),
$$

with

$$
\hat{\mathcal{E}}(\hat{\boldsymbol{\theta}}) \equiv-\frac{2 \varepsilon_{0} c \lambda}{\gamma e} \mathcal{E}_{\omega}(\boldsymbol{\theta}, 0)=\frac{1}{\gamma \lambda} \int\left[\beta_{x, y}(z)-\theta_{x, y}\right] \mathrm{e}^{i \omega \tau(z)} d z,
$$

being the normalized complex amplitude at the source point. Note that the normalization factors should have the relation 


$$
\chi \chi^{\prime}=\frac{\lambda}{2 \pi},
$$

so that the definition of $\hat{W}$ is consistent with the definition of Wigner function.

In order to define the relation between the phase-space density $d$ and the normalized Wigner function $\hat{W}$, let us consider the total photon flux, i.e., the number of photons in a unit bandwidth

$$
\frac{d N}{d \omega / \omega}=\iint d(\boldsymbol{\rho}, \boldsymbol{\theta}) d \boldsymbol{\rho} d \boldsymbol{\theta}
$$

which is also calculated by

$$
\begin{aligned}
\frac{d N}{d \omega / \omega} & =\frac{\varepsilon_{0} c \lambda^{2}}{\pi \hbar} \int|\mathcal{E}(\boldsymbol{\theta})|^{2} d \boldsymbol{\theta} \\
& =\frac{\alpha \gamma^{2}}{4 \pi^{2} \chi^{\prime 2}} \iint \hat{W}(\hat{\boldsymbol{\rho}}, \hat{\boldsymbol{\theta}}) d \hat{\boldsymbol{\rho}} d \hat{\boldsymbol{\theta}},
\end{aligned}
$$

where $\hbar$ is the reduced Planck's constant and $\alpha$ the fine structure constant. Comparing the two formulas above, we finally have

$$
d=\frac{\alpha \gamma^{2}}{\lambda^{2} \chi^{\prime 2}} \hat{W}
$$

\section{Convolution with the electron beam distribution function}

The discussions so far have been limited to the special case of SR emitted from a single electron. In the case of the practical electron beam, we have to take into account the effects due to the finite emittance and energy spread. To be more specific, the normalized Wigner function is given by

$$
\hat{W}(\hat{\boldsymbol{\rho}}, \hat{\boldsymbol{\theta}})=\iiint \hat{W}_{s}\left(\hat{\boldsymbol{\rho}}-\hat{\boldsymbol{\rho}}^{\prime}, \hat{\boldsymbol{\theta}}-\hat{\boldsymbol{\theta}}^{\prime}, \eta\right) p\left(\hat{\boldsymbol{\rho}}^{\prime}, \hat{\boldsymbol{\theta}}^{\prime}, \eta\right) d \hat{\boldsymbol{\rho}}^{\prime} d \hat{\boldsymbol{\theta}}^{\prime} d \eta,
$$

where $\eta=\left(\gamma-\gamma_{0}\right) / \gamma_{0}$ denotes the normalized energy deviation from the average $\gamma_{0}, \hat{W}_{s}$ the normalized Wigner function for a single electron defined in Eq. (9) with the argument $\eta$ being explicitly included, and $p$ the distribution function of the electron beam represented in the $(\hat{\boldsymbol{\rho}}, \hat{\boldsymbol{\theta}}, \eta)$ phase space. In derivation of Eq. (13), we have made an assumption that the magnetic field of the SR source does not depend on the transverse position. As a result, effects due to the positional and angular offsets of each electron are taken into account by the convolution integral with respect to $\hat{\boldsymbol{\rho}}^{\prime}$ and $\hat{\boldsymbol{\theta}}^{\prime}$ [6]. Other special cases when this is not validated, such as SR sources with focusing magnets, including the natural focusing of insertion devices, are out of the scope of this paper.
Now let us assume that the distribution function $p$ can be decoupled to Gaussian functions, i.e.,

$$
p(U, V, u, v, \eta)=f(U, u) g(V, v) h(\eta),
$$

with

$$
\begin{aligned}
f(U, u) & =\frac{1}{2 \pi \sigma_{U} \sigma_{u}} \exp \left[-\frac{\left(U+\hat{\alpha}_{u}\right)^{2}}{2 \sigma_{U}}-\frac{u^{2}}{2 \sigma_{u}^{2}}\right], \\
g(V, v) & =\frac{1}{2 \pi \sigma_{V} \sigma_{v}} \exp \left[-\frac{\left(V+\hat{\alpha}_{v}\right)^{2}}{2 \sigma_{V}}-\frac{v^{2}}{2 \sigma_{v}^{2}}\right], \\
h(\eta) & =\frac{1}{\sqrt{2 \pi} \sigma_{\eta}} \exp \left(-\frac{\eta^{2}}{2 \sigma_{\eta}^{2}}\right),
\end{aligned}
$$

which is usually the case for the electron beam in SR facilities. In the above formulas, we have introduced several parameters to specify the electron beam quality. The meaning of $\sigma_{\eta}$ is straightforward. As for the other parameters in Eqs. (15) and (16), refer to the Appendix about how they are related with the typical beam parameters such as the emittance, Twiss parameters, and dispersion functions.

Let us now turn to the convolution integral. In the $(U, u)$ plane, we have

$$
\begin{aligned}
& \iint \hat{W}_{s}\left(U-U^{\prime}, u-u^{\prime}\right) f\left(U^{\prime}, u^{\prime}\right) d U^{\prime} d u^{\prime} \\
& =\int \mathrm{e}^{-i u^{\prime \prime} U} d u^{\prime \prime} \int \mathrm{e}^{i u^{\prime \prime} U^{\prime}} d U^{\prime} \int \mathcal{W}\left(u-u^{\prime}, u^{\prime \prime}\right) f\left(U^{\prime}, u^{\prime}\right) d u^{\prime} \\
& =\frac{1}{\sqrt{2 \pi} \sigma_{u}} \int \exp \left(-i u^{\prime \prime} U-\frac{\sigma_{U}^{2} u^{\prime \prime 2}}{2}\right) d u^{\prime \prime} \\
& \quad \times \int \mathcal{W}\left(u-u^{\prime}, u^{\prime \prime}\right) \exp \left(-i \hat{\alpha}_{u} u^{\prime} u^{\prime \prime}-\frac{u^{\prime 2}}{2 \sigma_{u}^{2}}\right) d u^{\prime},
\end{aligned}
$$

where we have omitted several arguments that are not relevant. Note that the integration over $U^{\prime}$ is reduced to a simple Fourier transform of a Gaussian function by substituting Eq. (15), and has been carried out analytically. The convolution in the $(V, v)$ plane can be done in the same manner. The normalized Wigner function for the practical electron beam is thus given by

$$
\begin{aligned}
\hat{W}(\hat{\boldsymbol{\rho}}, \hat{\boldsymbol{\theta}})= & \frac{1}{2 \pi \sigma_{u} \sigma_{v}} \int \exp \left(-i \hat{\boldsymbol{\theta}}^{\prime \prime} \cdot \hat{\boldsymbol{\rho}}-\frac{\sigma_{U}^{2} u^{\prime \prime 2}+\sigma_{V}^{2} v^{\prime \prime 2}}{2}\right) d \hat{\boldsymbol{\theta}}^{\prime \prime} \\
& \times \int \exp \left(-i \hat{\alpha}_{u} u^{\prime} u^{\prime \prime}-i \hat{\alpha}_{v} v^{\prime} v^{\prime \prime}-\frac{u^{\prime 2}}{2 \sigma_{u}^{2}}-\frac{v^{\prime 2}}{2 \sigma_{v}^{2}}\right) d \hat{\boldsymbol{\theta}}^{\prime} \\
& \times \int h(\eta) \mathcal{W}\left(\hat{\boldsymbol{\theta}}-\hat{\boldsymbol{\theta}}^{\prime}, \hat{\boldsymbol{\theta}}^{\prime \prime}, \eta\right) d \eta .
\end{aligned}
$$

The integration over $\eta$ in Eq. (17) means the convolution with the energy distribution function of the electron beam 
to take into account the effects due to the energy spread. It is easy to understand that this integration can be omitted for BMR and WR, because the complex amplitudes of them are not sensitive to the electron energy $\gamma$, as explained later. This is not the case for UR whose spectrum has a sharp peak depending on $\gamma$, and thus the numerical cost to evaluate $\hat{W}$ can be much higher than those of BMR and WR, when the energy spread is not negligible. This problem is discussed later in more detail, together with a countermeasure against it.

\section{SPECIFIC FORMULAS FOR TYPICAL SR SOURCES}

Equation (17) is the universal formula to compute the phase-space density of SR from the practical electron beam. Now let us actually apply this formula to three typical SR sources: BMs, undulators, and wigglers.

\section{A. Bending magnets}

The relative velocity of an electron passing through the uniform field generated by a BM is given as

$$
\beta_{x}(z)=\frac{z}{R_{b}}, \quad \beta_{y}(z)=0,
$$

where $R_{b}$ denotes the bending radius. Substituting into Eq. (10), we have the normalized complex amplitude of BMR for the horizontally polarized component as follows

$$
\begin{aligned}
\hat{\mathcal{E}} & =\frac{1}{\gamma \lambda} \int\left(\frac{z}{R_{b}}-\theta_{x}\right) \exp \left\{\frac{i k}{2 \gamma^{2}} \int_{0}^{z}\left[1+\gamma^{2} \theta_{y}^{2}+\left(\gamma \theta_{x}-\frac{\gamma z^{\prime}}{R_{b}}\right)^{2}\right] d z^{\prime}\right\} d z \\
& =\frac{1}{\gamma \lambda} \mathrm{e}^{i \Lambda} \int \frac{\bar{z}}{R_{b}} \exp \left\{\frac{i k}{2 \gamma^{2}}\left[\left(1+\gamma^{2} \theta_{y}^{2}\right) \bar{z}+\frac{\gamma^{2} \bar{z}^{3}}{3 R_{b}^{2}}\right]\right\} d \bar{z} .
\end{aligned}
$$

with

$$
\Lambda=\frac{k R_{b}}{2 \gamma^{2}} \theta_{x}\left(1+\gamma^{2} \theta_{y}^{2}+\frac{\gamma^{2} \theta_{x}^{2}}{3}\right),
$$

being the phase of radiation.

It is well known that the integration can be done analytically and be given by the modified Bessel functions [14]. We introduce the angular normalization factor defined as

$$
\chi^{\prime}=\frac{1}{\epsilon \gamma},
$$

with

$$
\epsilon=\left(\frac{3 \omega}{4 \omega_{c}}\right)^{1 / 3}
$$

being the normalized photon energy, where $\omega_{c}$ denotes the critical frequency of BMR. Then we have

$$
\hat{\mathcal{E}}(\hat{\boldsymbol{\theta}})=\frac{2 i}{\sqrt{3} \pi} \epsilon\left(\epsilon^{2}+v^{2}\right) K_{2 / 3}\left[\frac{2}{3}\left(\epsilon^{2}+v^{2}\right)^{3 / 2}\right] \mathrm{e}^{i \Lambda},
$$

with

$$
\Lambda=\epsilon u\left(\epsilon^{2}+v^{2}+\frac{u^{2}}{3}\right)
$$

Note that the positional normalization factor is defined as

$$
\chi=\frac{R_{b}}{2 \epsilon^{2} \gamma^{2}},
$$

to satisfy the relation (11). In the same manner, we have

$\hat{\mathcal{E}}(\hat{\boldsymbol{\theta}})=-\frac{2}{\sqrt{3} \pi} \epsilon v \sqrt{\epsilon^{2}+v^{2}} K_{1 / 3}\left[\frac{2}{3}\left(\epsilon^{2}+v^{2}\right)^{3 / 2}\right] \mathrm{e}^{i \Lambda}$,

for the vertically polarized component. Using Eqs. (9), (19), and (20), the normalized Wigner function $\hat{W}$ can be computed for BMR emitted by a single electron.

Figure 1(a) shows the distribution of $\hat{W}$ at the critical frequency $\left(\omega=\omega_{c}\right)$ plotted in the horizontal phase space $(U, u)$, where the vertical coordinates have been assumed to be $0(V=v=0)$. We find a profile composed of many layers, each of which has a parabolic shape. It should be noted, however, that this multilayer structure easily disappears under a practical condition as shown in Fig. 1(b), in which $\hat{W}$ has been computed under the same condition as in Fig. 1(a) except that $\sigma_{U}=1$ has been assumed. This corresponds to the horizontal beam size of $0.12 \mu \mathrm{m}$, if we assume the electron energy of $6 \mathrm{GeV}$ and bending radius of $30 \mathrm{~m}$, which is much smaller than typical values in practical accelerators for SR sources. We find that with such a small but finite beam size, all the layers but the main one have disappeared.

The parabolic shape of $\hat{W}$ in the horizontal phase space can be qualitatively explained by the geometrical optics using Fig. 2, in which an electron moving along a circular orbit with a radius of $R_{b}$ is schematically illustrated. Let us assume that a photon is emitted toward the tangential 


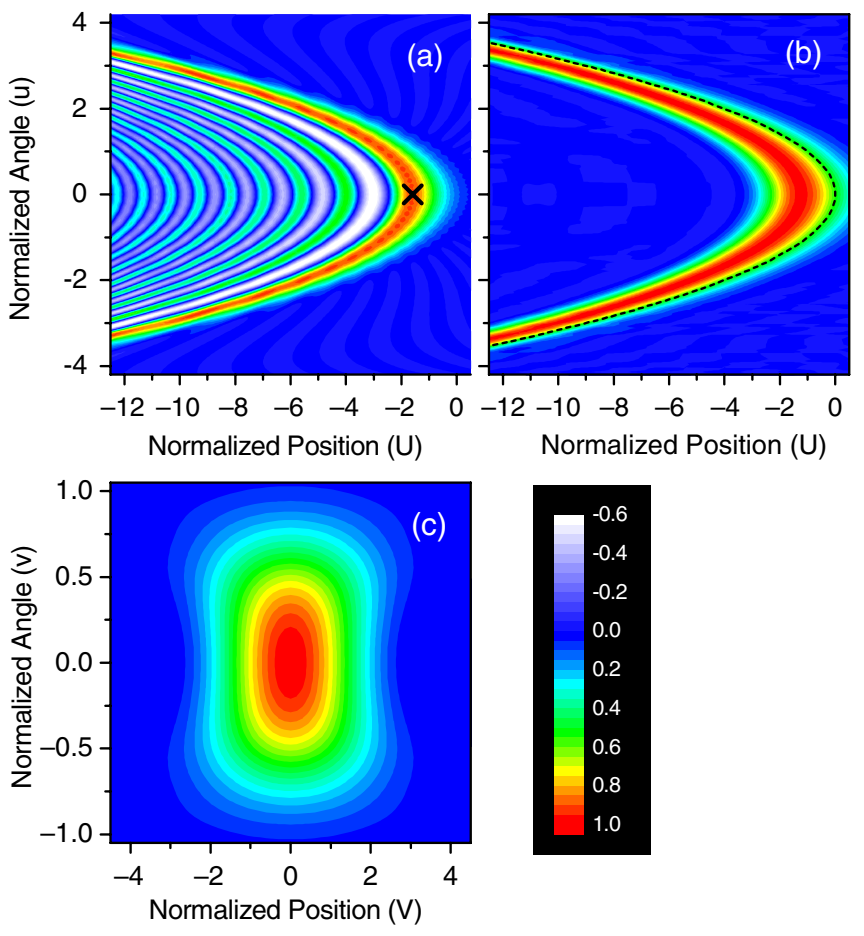

FIG. 1. Normalized Wigner function of BMR emitted by a single electron calculated on the $(U, u)$ plane sliced at $(V, v)=(0,0)$. (b) Same as (a), but $\sigma_{U}=1$ has been assumed to investigate the effects due to the finite electron beam size in the horizontal direction. The dashed line shows the graphical plot of $U=-u^{2}$ (refer to the text). (c) Same as (a), but on the $(V, v)$ plane sliced at $(U, u)=(-1.6,0)$.

direction, when the electron passes through the point A. Then it is easy to show that the horizontal coordinate $X$ of the photon at the longitudinal origin $(z=0)$ is given by $X=-R_{b} \theta_{x}^{2} / 2$. Using the normalization factors $\chi$ and $\chi^{\prime}$, this reduces to a relation $U=-u^{2}$. Thus the photon distribution in the horizontal phase space is dominated

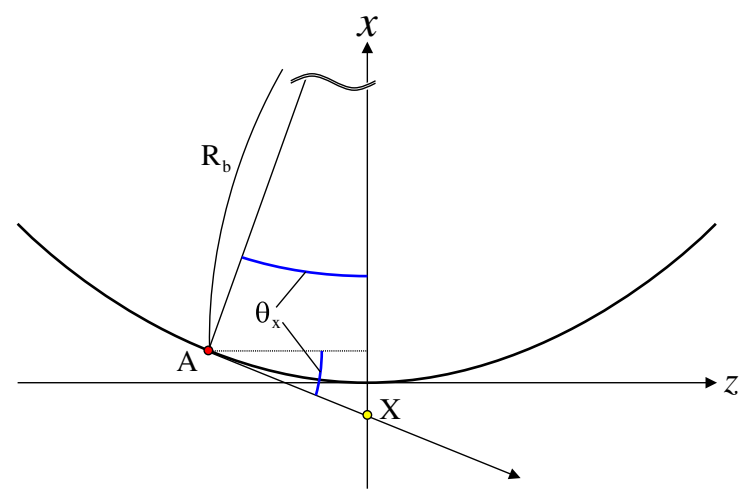

FIG. 2. Schematic illustration of emission of radiation from an electron (red circle) moving along the circular orbit indicated by the solid black line. The coordinate $X$ denotes the horizontal position of a photon (yellow circle) at the longitudinal origin, which is emitted from the electron when it passes the point A. by this function, which is actually shown by the dashed line in Fig. 1(b).

Figure 1(c) shows the distribution of $\hat{W}$ as in Fig. 1(a), but in the vertical phase space $(V, v)$, where the horizontal coordinates have been assumed to be $(U, u)=(-1.6,0)$ as indicated by the black " $x$ " in (a). Compared to Fig. 1(a) or (b), the profile looks more similar to that of the Gaussian beam.

\section{B. Undulators}

The electron trajectory in an undulator is characterized by the periodic condition given as

$$
\boldsymbol{\beta}\left(z+\lambda_{u}\right)=\boldsymbol{\beta}(z),
$$

where $\lambda_{u}$ is the undulator period. Assuming the number of periods to be $N$, the normalized complex amplitude of UR is given as

$$
\hat{\mathcal{E}}=\frac{1}{\lambda \gamma^{2}} \int_{-N T / 2}^{N T / 2} f(\tau) \mathrm{e}^{i \omega \tau} d \tau,
$$

with

$$
f(\tau)=\left(\gamma \beta_{x, y}-\gamma \theta_{x, y}\right) \frac{d z}{d \tau},
$$

being a periodic function of $\tau$ with the period of $T$. Note that the integration variable in Eq. (10) has been changed from $z$ to $\tau$. It is well known that the period $T$ depends on $\gamma$ and $\boldsymbol{\theta}$, and is given by

$$
T(\gamma, \boldsymbol{\theta})=\frac{\left(1+\gamma^{2} \theta^{2}+K_{x}^{2} / 2+K_{y}^{2} / 2\right) \lambda_{u}}{2 \gamma^{2} c},
$$

where $K_{x}$ and $K_{y}$ are the horizontal and vertical deflection parameters of the undulator, respectively. The frequency defined by $\omega_{1}(\gamma, \boldsymbol{\theta})=2 \pi / T(\gamma, \boldsymbol{\theta})$ is referred to as the fundamental frequency of UR, at which the spectrum has a sharp peak.

Expanding the periodic function $f(\tau)$ into a Fourier series, we have

$$
\hat{\mathcal{E}}=\frac{1}{\lambda \gamma^{2}} \int_{-N T / 2}^{N T / 2}\left(\sum_{n} f_{n} \mathrm{e}^{-i n \omega_{1} \tau}\right) \mathrm{e}^{i \omega \tau} d \tau
$$

where $f_{n}$ denotes the Fourier coefficient. Then the integration in Eq. (21) can be done analytically and $\hat{\mathcal{E}}$ is represented as a summation of harmonics, i.e.,

$$
\hat{\mathcal{E}}=\frac{N \lambda_{u}}{\lambda \gamma^{2}} \sum_{n} \hat{f}_{n} S_{n}
$$

with 


$$
\hat{f}_{n} \equiv \frac{T f_{n}}{\lambda_{u}}=\frac{1}{\lambda_{u}} \int_{0}^{\lambda_{u}}\left(\gamma \beta_{x, y}-\gamma \theta_{x, y}\right) \mathrm{e}^{i n \omega_{1} \tau(z)} d z
$$

and

$$
S_{n}=\operatorname{sinc}\left(\pi n N \frac{\omega-n \omega_{1}}{n \omega_{1}}\right)
$$

where we have introduced the so-called sinc function defined as

$$
\operatorname{sinc}(x) \equiv \sin (x) / x
$$

Note that $f_{n}$ denotes the complex amplitude of radiation generated within a single period at the $n$th harmonic photon energy, while $S_{n}$ denotes its enhancement by coherent sum of radiation over $N$ periods.

For further discussions, let us introduce the reference electron energy $\gamma_{0}$, which usually refers to the average energy of the electron beam. Then $\omega_{0}=2 \pi / T\left(\gamma_{0}, 0\right)$ and $\lambda_{0}=2 \pi c / \omega_{0}$ denote the corresponding fundamental frequency and wavelength observed on axis, respectively. We then define the angular normalization factor $\chi^{\prime}=2 \sigma_{n r^{\prime}}$, where $\sigma_{n r^{\prime}}$ is the angular divergence of UR at the $n$th harmonic defined by

$$
\sigma_{n r^{\prime}}=\sqrt{\frac{\lambda_{0}}{2 n L}}
$$

where $L$ denotes the undulator length. This equation has been derived by a Gaussian approximation of $|\mathcal{E}|^{2}$, i.e., the angular profile of UR [6]. It should be noted that the above approximation is correct only at $n \omega_{0}$, the $n$th harmonic photon energy, and the angular divergence at other detuned energies cannot be clearly defined.

It is easy to show that the positional normalization factor is given by $\chi=\sigma_{n r}$, where $\sigma_{n r}$ denotes the source size of UR defined by

$$
\sigma_{n r}=\frac{\lambda}{4 \pi n \sigma_{n r^{\prime}}},
$$

which is derived by assuming that UR emitted by a single electron is diffraction limited.

Equation (22) now reduces to

$$
S_{n}(\hat{\theta}, \eta)=\operatorname{sinc}\left[\pi \hat{\theta}^{2}\left(1+\frac{\epsilon}{n N}\right)+\pi \epsilon\right],
$$

with

$$
\epsilon=n N\left(\frac{\omega-n \omega_{0}}{n \omega_{0}}-2 \eta\right)
$$

which denotes the deviation of the electron and photon energies from the reference values $\gamma_{0}$ and $\hbar \omega_{0}$.

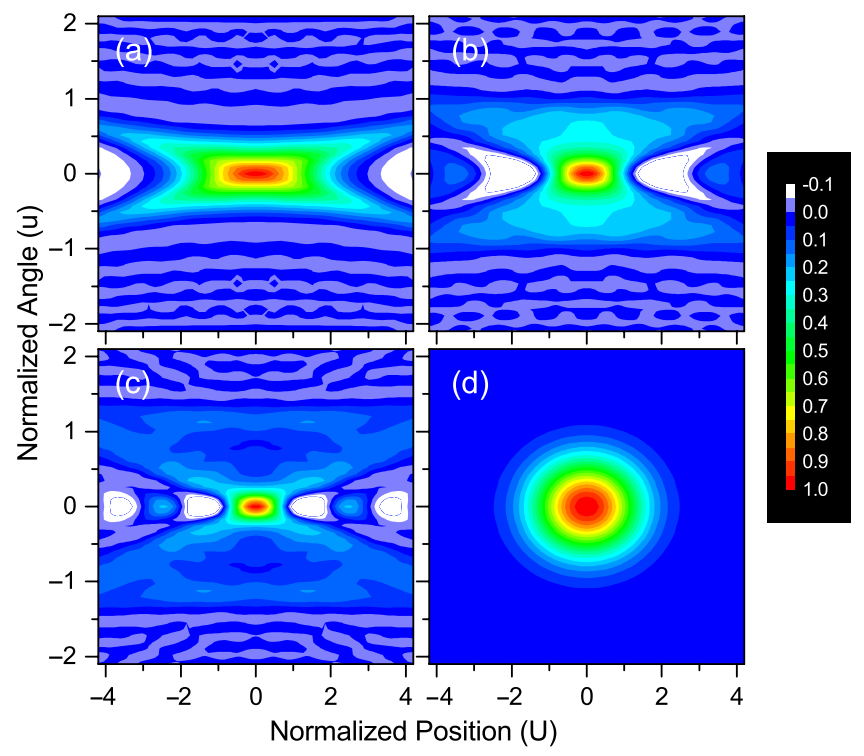

FIG. 3. Normalized Wigner function of UR emitted by a single electron plotted on the $(U, u)$ plane sliced at $(V, v)=(0,0)$ for three different energy deviations: (a) $\epsilon=0$, (b) $\epsilon=-1$, and (c) $\epsilon=-2$. The 2D Gaussian profile with the standard deviation of $\sigma_{U}=1$ and $\sigma_{u}=1 / 2$ is plotted in (d) for reference.

Figures 3(a)-(c) show the Wigner functions of the fundamental UR plotted in the $(U, u)$ plane, for three different values of energy deviation: (a) $\epsilon=0$, (b) $\epsilon=-1$, and (c) $\epsilon=-2$. Note that the vertical coordinates have been assumed to be $0(V=v=0)$ and individual Wigner functions are normalized by the maximum values. It is found that the distribution shrinks more for smaller values of $\epsilon$.

From the above discussions, together with the definition of $\epsilon$ (24), it is easy to understand that we cannot omit the integration over $\eta$ in Eq. (17), which increases the total number of numerical operations by at least one order of magnitude. Its numerical cost, however, can be made nearly negligible by means of a numerical technique explained below.

The complex amplitude of the $n$th harmonic is given as a product of two functions $f_{n}$ and $S_{n}$ except the constants. It is easy to understand that the latter changes rapidly as $\gamma$ especially around $\gamma_{0}$, while the former is a slowly varying function of $\gamma$. It is thus reasonable to factor $f_{n}$ out of the integral. To be more specific, we have

$$
\begin{aligned}
\int h(\eta) \mathcal{W}\left(\hat{\boldsymbol{\theta}}-\hat{\boldsymbol{\theta}}^{\prime}, \hat{\boldsymbol{\theta}}^{\prime \prime}, \eta\right) d \eta \propto \int h(\eta) S_{n}\left(\hat{\theta}_{1}, \eta\right) S_{n}\left(\hat{\theta}_{2}, \eta\right) d \eta \\
\equiv P_{n}\left(\hat{\theta}_{1}, \hat{\theta}_{2}\right)
\end{aligned}
$$

with

$$
\hat{\boldsymbol{\theta}}_{1,2}=\hat{\boldsymbol{\theta}}-\hat{\boldsymbol{\theta}}^{\prime} \pm \frac{\hat{\boldsymbol{\theta}}^{\prime \prime}}{2}
$$


where the function $P_{n}$ can be computed easily once the parameters such as $n, N$, and $\sigma_{\eta}$ are given. In order to reduce the numerical cost, we create the tabulated data of $P_{n}$ in advance of starting the numerical computation of Eq. (17), which can be used to interpolate at arbitrary arguments of $\hat{\boldsymbol{\theta}}_{1,2}$. The interval and range of the tabulated data should be decided so that the interpolation of $P_{n}$ is accurate enough, but its numerical cost and required memory size are not too large. In practice, our experiences show that the numerical cost of creating the tabulated data with enough data points is much less than that of other numerical operations.

It is interesting to compare the results shown in Figs. 3(a)-(c) with the conventional results based on the Gaussian approximation as shown in Fig. 3(d), where a 2D Gaussian function is plotted, with the standard deviations of 1 and $1 / 2$ in the $U$ and $u$ directions, respectively. This corresponds to the Gaussian beam having the source size of $\sigma_{1 r}$ and angular divergence of $\sigma_{1 r^{\prime}}$. The difference between the two distribution functions based on the Wigner function and the Gaussian approximation leads to the difference in the optimum betatron function to be discussed later.

\section{Wigglers}

Wigglers are equivalent to undulators in terms of the magnetic circuit. Namely, both devices generate a periodic magnetic field to enhance the radiation intensity. The properties of WR, however, differs significantly from those of UR, because the radiation is incoherently summed up over the whole periods. The calculation of the Wigner function should be done accordingly.

If we assume that the wiggler is composed of many small BMs with the alternating polarity, it is reasonable to calculate the Wigner function of WR by summing up all the local Wigner functions that correspond to individual half periods (magnetic poles), the procedure of which is explained in the following sections. Note that each small BM should have a field gradient along the longitudinal axis, which corresponds to the sinusoidal field distribution of the wiggler.

\section{Complex amplitude of radiation generated in the central magnetic pole}

The electron trajectory in a wiggler is given as

$$
\beta_{x}(z)=\frac{K}{\gamma} \sin k_{u} z, \beta_{y}(z)=0,
$$

where $\lambda_{u}=2 \pi / k_{u}$ denotes the wiggler period and $K$ denotes the deflection parameter. Note that the polarity of the magnet pole positioned at the center of the wiggler is the same as that of the BM discussed in Sec. III A, which is defined to be positive. The normalized complex amplitude of radiation for the horizontally polarized component, which is generated in this magnetic pole, is given as

$$
\hat{\mathcal{E}}_{0}^{+}(\hat{\boldsymbol{\theta}})=\frac{1}{\gamma^{2} \lambda} \int_{-\lambda_{u} / 4}^{\lambda_{u} / 4}\left(K \sin k_{u} z-\gamma \theta_{x}\right) \mathrm{e}^{i \omega \tau(z)} d z
$$

with

$$
\tau(z)=\frac{1}{2 \gamma^{2} c} \int^{z}\left[1+\gamma^{2} \theta_{y}^{2}+\left(K \sin k_{u} z^{\prime}-\gamma \theta_{x}\right)^{2}\right] d z^{\prime} .
$$

The integration in Eq. (26) can be done semianalytically by means of an approximate method following the formulation of BMR as explained below.

First we change the integration variable from $z$ to $\bar{z}=z-\phi_{0} / k_{u}$, where $\phi_{0}$ is an angle to satisfy the two conditions: $\left|\phi_{0}\right| \leq \pi / 2$ and $\sin \phi_{0}=\gamma \theta_{x} / K$. Obviously, $\phi_{0}$ cannot be defined when $\left|\theta_{x}\right|>K / \gamma$, in which case $\hat{\mathcal{E}}_{0}^{+}$is defined to be zero. Otherwise, Eq. (26) reduces to

$$
\begin{aligned}
\hat{\mathcal{E}}_{0}^{+}(\hat{\boldsymbol{\theta}})= & \frac{K}{\gamma^{2} \lambda} \mathrm{e}^{i \Lambda} \int q(\bar{z}) \\
& \times \exp \left\{\frac{i k}{2 \gamma^{2}} \int_{0}^{\bar{z}}\left[1+\gamma^{2} \theta_{y}^{2}+p^{2}\left(\bar{z}^{\prime}\right)\right] d \bar{z}^{\prime}\right\} d \bar{z},
\end{aligned}
$$

with

$$
q(\bar{z})=2 \sin \left(\frac{k_{u} \bar{z}}{2}\right) \cos \left(\frac{k_{u} \bar{z}}{2}+\phi_{0}\right)
$$

and

$$
\begin{aligned}
\Lambda= & \frac{k}{2 \gamma^{2} k_{u}}\left[\left(1+\gamma^{2} \theta^{2}+K^{2} / 2\right) \phi_{0}\right. \\
& \left.+\gamma \theta_{x}\left(\frac{3}{2} \sqrt{K^{2}-\gamma^{2} \theta_{x}^{2}}-2\right)\right] .
\end{aligned}
$$

Because a small portion of the electron trajectory around the longitudinal position $z=\phi_{0} / k_{u} \equiv z_{0}$ contributes to the formation of radiation observed at the horizontal angle $\theta_{x}$, the integration in the above equation converges within a narrow range $\left|k_{u} \bar{z}\right| \sim 0$. Thus the function $q$ can be replaced as

$$
q(\bar{z}) \rightarrow k_{u} \bar{z} \cos k_{u} z_{0},
$$

with which Eq. (27) reduces to

$$
\begin{aligned}
\hat{\mathcal{E}}_{0}^{+}(\hat{\boldsymbol{\theta}})= & \frac{1}{\gamma \lambda} \mathrm{e}^{i \Lambda} \int \frac{\bar{z}}{R_{b}\left(\theta_{x}\right)} \\
& \times \exp \left\{\frac{i k}{2 \gamma^{2}}\left[\left(1+\gamma^{2} \theta_{y}^{2}\right) \bar{z}+\frac{\gamma^{2} \bar{z}^{3}}{3 R_{b}^{2}\left(\theta_{x}\right)}\right]\right\} d \bar{z},
\end{aligned}
$$

with

$$
R_{b}\left(\theta_{x}\right)=\frac{\gamma}{k_{u} K \sqrt{1-\left(\gamma \theta_{x} / K\right)^{2}}},
$$

being the bending radius at the longitudinal position $z=z_{0}$. We now find that Eq. (28) is similar to (18) and thus the integration can be done analytically. 
As in the case of BMR, we introduce the normalization factors as follows

$$
\epsilon=\left(\frac{3 \omega}{4 \omega_{c}}\right)^{1 / 3}, \quad \chi^{\prime}=\frac{1}{\epsilon \gamma},
$$

with

$$
\omega_{c}=\frac{3 \pi \gamma^{2} c K}{\lambda_{u}},
$$

being the critical frequency corresponding to the maximum wiggler field. The positional normalization factor $\chi$ can be modified as

$$
\chi=\frac{K}{\gamma k_{u}} \frac{1}{2 \epsilon^{2} K^{2}} \equiv \frac{x_{a}}{2 \epsilon^{2} K^{2}}
$$

where $x_{a}$ denotes the amplitude of the electron trajectory.

Using the normalized angular coordinate, $\hat{\mathcal{E}}_{0}^{+}$is given as

$$
\hat{\mathcal{E}}_{0}^{+}(\hat{\boldsymbol{\theta}})=\frac{2 i}{\sqrt{3} \pi} \frac{\epsilon\left(\epsilon^{2}+v^{2}\right)}{\sqrt{1-(u / \epsilon K)^{2}}} K_{2 / 3}\left[\frac{2\left(\epsilon^{2}+v^{2}\right)^{3 / 2}}{3 \sqrt{1-(u / \epsilon K)^{2}}}\right] \mathrm{e}^{i \Lambda},
$$

with

$$
\begin{aligned}
\Lambda= & \epsilon K\left[\left(\epsilon^{2}+u^{2}+v^{2}+\frac{\epsilon^{2} K^{2}}{2}\right) \sin ^{-1}\left(\frac{u}{\epsilon K}\right)\right. \\
& \left.+u\left(\frac{3}{2} \sqrt{\epsilon^{2} K^{2}-u^{2}}-2\right)\right] .
\end{aligned}
$$

In the same manner, we have

$\hat{\mathcal{E}}_{0}^{+}(\hat{\boldsymbol{\theta}})=-\frac{2}{\sqrt{3} \pi} \frac{\epsilon v \sqrt{\epsilon^{2}+v^{2}}}{\sqrt{1-(u / \epsilon K)^{2}}} K_{1 / 3}\left[\frac{2\left(\epsilon^{2}+v^{2}\right)^{3 / 2}}{3 \sqrt{1-(u / \epsilon K)^{2}}}\right] \mathrm{e}^{i \Lambda}$,

for the vertically polarized component.

In order to check the validity of the above method, $\hat{\mathcal{E}}_{0}^{+}$ has been computed with the semianalytical formula (29) and compared with the more accurate numerical results based on Eq. (26), which is shown in Fig. 4. The absolute value and argument of $\hat{\mathcal{E}}_{0}^{+}$computed with (29) are plotted as a function of $u$, together with the deviations from the rigorous results with (26). Note that $K=10, v=0$, and $\omega=\omega_{c}$ have been assumed. We find that the deviations are negligible nearly in the whole range of interest, except around the angle indicated by the dotted line, which corresponds to $\left|\theta_{x}\right|=K / \gamma$. This angle is usually much larger than $\gamma^{-1}$, because the deflection parameter of any wiggler is much larger than unity. Thus the application of semianalytical formula (29) is validated as long as we are

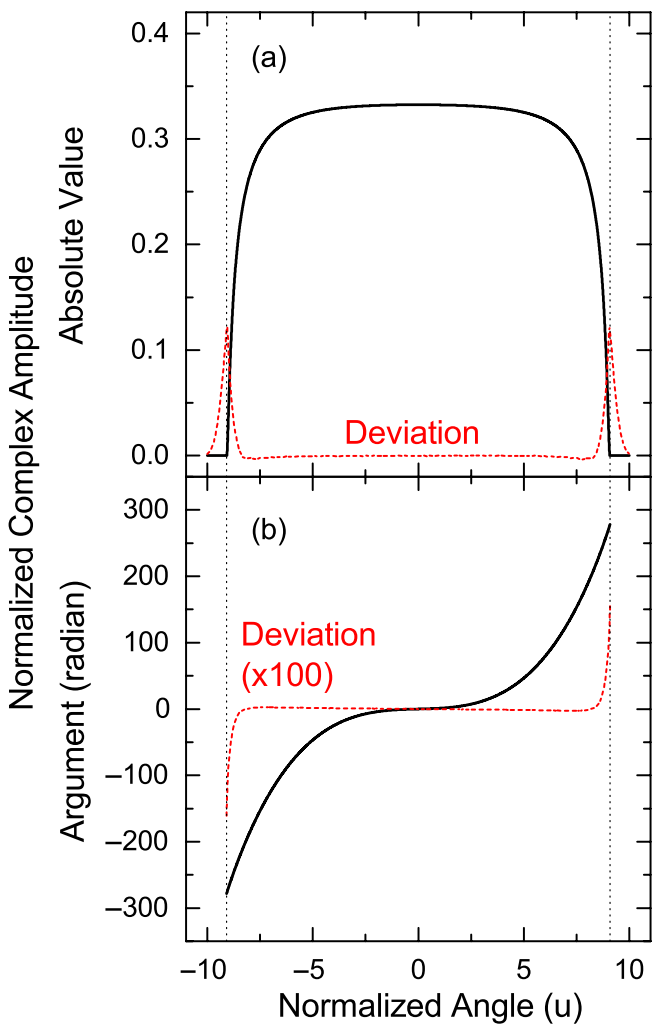

FIG. 4. Complex amplitude $\hat{\mathcal{E}}_{0}$ as a function of the horizontal angle $u$ in terms of (a) absolute value and (b) argument. The black solid line shows the semianalytical results using Eq. (29), while the red dashed lines show deviations from the more accurate numerical results using Eq. (26).

not interested in characterization of radiation in such a large angle region.

The above discussions for the positive pole can be extended to calculate the complex amplitude $\hat{\mathcal{E}}_{0}^{-}$of radiation generated in the negative pole positioned at the wiggler center. By reversing the sign of $\beta_{x}$ in Eq. (25), it is easy to show

$$
\hat{\mathcal{E}}_{0}^{-}(\hat{\boldsymbol{\theta}})=-\hat{\mathcal{E}}_{0}^{+*}(\hat{\boldsymbol{\theta}})
$$

\section{Summation of local Wigner functions}

The complex amplitude $\hat{\mathcal{E}}_{0}^{ \pm}(\hat{\boldsymbol{\theta}})$ derived in the previous section can be used to compute the local Wigner function at the central magnetic pole. Next we extend this procedure to other magnetic poles positioned away from the origin, and sum up all the local Wigner functions over the whole periods.

Assuming that the wiggler magnet starts with the positive pole, the longitudinal positions of the $m$ th positive and negative poles are given as

$$
z_{m}^{ \pm}=\left(m-\frac{N+1}{2} \pm \frac{1}{4}\right) \lambda_{u} \equiv \nu_{m}^{ \pm} \lambda_{u}
$$


where the superscript \pm stands for the polarity of the magnetic pole. The complex amplitude of radiation, which is generated at the $m$ th positive pole and is propagated to the wiggler origin, is obtained by multiplying $\hat{\mathcal{E}}_{0}^{+}$by the phase factor describing the diffraction effect. Namely, we have

$$
\hat{\mathcal{E}}_{m}^{+}=\hat{\mathcal{E}}_{0}^{+} \exp \left(i k z_{m}^{+} \boldsymbol{\theta}^{2} / 2\right)=\hat{\mathcal{E}}_{0}^{+} \exp \left(2 \pi i \nu_{m}^{+} \epsilon K \hat{\boldsymbol{\theta}}^{2}\right),
$$

with which the local Wigner function is calculated as

$$
\hat{W}_{m}^{+}(\hat{\boldsymbol{\rho}}, \hat{\boldsymbol{\theta}})=\int \mathcal{W}^{+}\left(\hat{\boldsymbol{\theta}}, \hat{\boldsymbol{\theta}}^{\prime}\right) \exp \left(-4 \pi i \nu_{m}^{+} \epsilon K \hat{\boldsymbol{\theta}} \cdot \hat{\boldsymbol{\theta}}^{\prime}\right) \mathrm{e}^{-i \hat{\boldsymbol{\theta}}^{\prime} \cdot \hat{\boldsymbol{\rho}}} d \hat{\boldsymbol{\theta}}^{\prime},
$$

with

$$
\mathcal{W}^{+}\left(\hat{\boldsymbol{\theta}}, \hat{\boldsymbol{\theta}}^{\prime}\right)=\hat{\mathcal{E}}_{0}^{+*}\left(\hat{\boldsymbol{\theta}}+\frac{\hat{\boldsymbol{\theta}}^{\prime}}{2}\right) \hat{\mathcal{E}}_{0}^{+}\left(\hat{\boldsymbol{\theta}}-\frac{\hat{\boldsymbol{\theta}}^{\prime}}{2}\right) .
$$

Summing up all the periods, the normalized Wigner function for the positive pole is given as

$$
\begin{aligned}
\hat{W}^{+}(\hat{\boldsymbol{\rho}}, \hat{\boldsymbol{\theta}})= & \sum_{m=1}^{N} \hat{W}_{m}^{+}(\hat{\boldsymbol{\rho}}, \hat{\boldsymbol{\theta}}) \\
= & \int \mathcal{W}^{+}\left(\hat{\boldsymbol{\theta}}, \hat{\boldsymbol{\theta}}^{\prime}\right) \exp \left(-\pi i \epsilon K \hat{\boldsymbol{\theta}} \cdot \hat{\boldsymbol{\theta}}^{\prime}\right) \\
& \times \frac{\sin \left(2 \pi \epsilon K N \hat{\boldsymbol{\theta}} \cdot \hat{\boldsymbol{\theta}}^{\prime}\right)}{\sin \left(2 \pi \epsilon K \hat{\boldsymbol{\theta}} \cdot \hat{\boldsymbol{\theta}}^{\prime}\right)} \mathrm{e}^{-i \hat{\boldsymbol{\theta}}^{\prime} \cdot \hat{\boldsymbol{\rho}}} d \hat{\boldsymbol{\theta}}^{\prime} .
\end{aligned}
$$

In the same manner, we have

$$
\begin{aligned}
\hat{W}^{-}(\hat{\boldsymbol{\rho}}, \hat{\boldsymbol{\theta}})= & \int \mathcal{W}^{+}\left(\hat{\boldsymbol{\theta}},-\hat{\boldsymbol{\theta}}^{\prime}\right) \exp \left(\pi i \epsilon K \hat{\boldsymbol{\theta}} \cdot \hat{\boldsymbol{\theta}}^{\prime}\right) \\
& \times \frac{\sin \left(2 \pi \epsilon K N \hat{\boldsymbol{\theta}} \cdot \hat{\boldsymbol{\theta}}^{\prime}\right)}{\sin \left(2 \pi \epsilon K \hat{\boldsymbol{\theta}} \cdot \hat{\boldsymbol{\theta}}^{\prime}\right)} \mathrm{e}^{-i \hat{\boldsymbol{\theta}}^{\prime} \cdot \hat{\boldsymbol{\rho}}} d \hat{\boldsymbol{\theta}}^{\prime} \\
= & \hat{W}^{+}(-\hat{\boldsymbol{\rho}}, \hat{\boldsymbol{\theta}}),
\end{aligned}
$$

for the negative pole. The normalized Wigner function of WR is now obtained by adding the two functions $\hat{W}^{+}$and $\hat{W}^{-}$. But before that, we need to recall the fact that the electron is shifted horizontally by a distance of $x_{a}$ alternately when it passes through the individual poles. This means that $\hat{W}^{+}$and $\hat{W}^{-}$should be translated along the $U$ axis by the distance of $x_{a} / \chi=2 \epsilon^{2} K^{2}$, but toward the opposite direction. Then we finally have the normalized Wigner function of WR as follows

$$
\begin{aligned}
\hat{W}(U, V, u, v)= & \hat{W}^{+}\left(U+2 \epsilon^{2} K^{2}, V, u, v\right) \\
& +\hat{W}^{+}\left(-U+2 \epsilon^{2} K^{2}, V, u, v\right) .
\end{aligned}
$$

Figure 5(a) shows the distribution of $\hat{W}$ at the critical frequency $\left(\omega=\omega_{c}\right)$ plotted in the horizontal phase space $(U, u)$, where $K=10, N=5$, and $(V, v)=(0,0)$ have
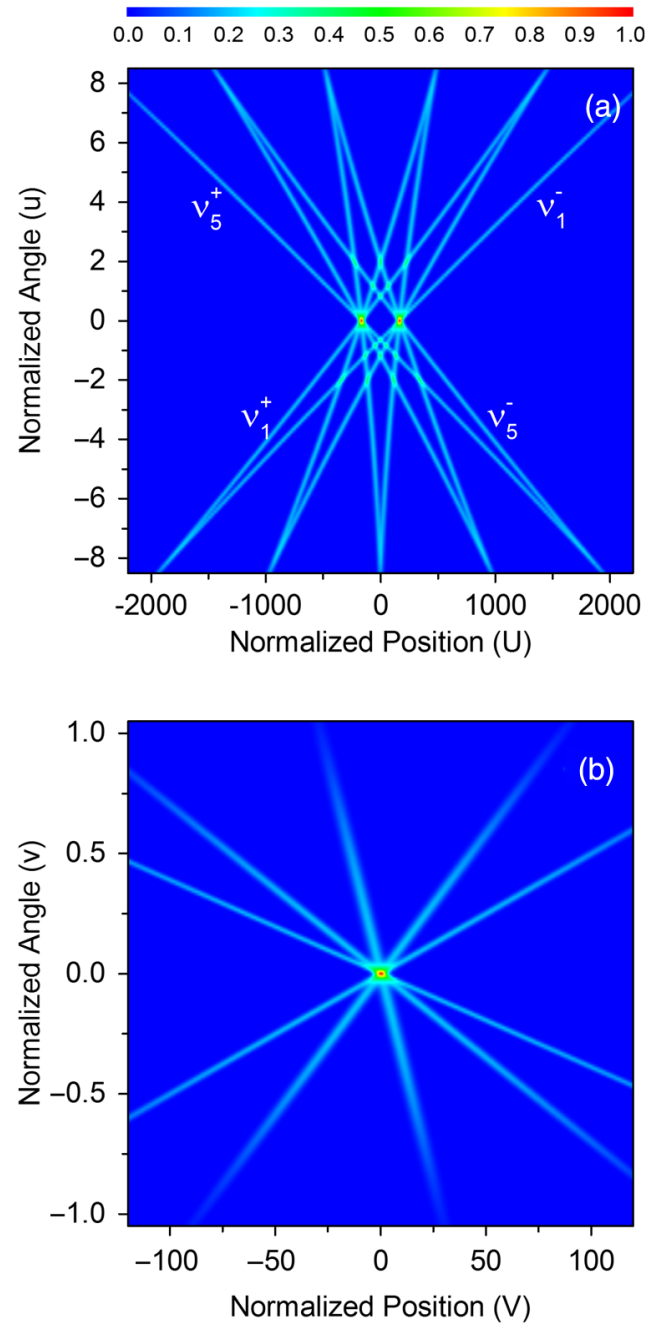

FIG. 5. Normalized Wigner function of WR plotted on (a) the $(U, u)$ plane sliced at $(V, v)=(0,0)$ and $(\mathrm{b})(V, v)$ plane sliced at $(U, u)=\left(-x_{a} / \chi, 0\right)$.

been assumed. We find 10 separate linear ridges, each of which corresponds to the magnetic pole distributed along the longitudinal axis. For example, the linear ridge indicated by $\nu_{1}^{-}$comes from the initial (1st) negative pole, while that by $\nu_{5}^{+}$from the final (5th) positive pole. We also find that the ridges coming from the negative poles intersect at the coordinates $(U, u)=\left(+x_{a} / \chi, 0\right)$, while those from the positive poles intersect at $(U, u)=\left(-x_{a} / \chi, 0\right)$. Obviously, this is attributed to the horizontal shift of the electron trajectory, $x_{a}$, described above. As a result, we have two source points in the horizontal direction in the case of WR, which has been already pointed out by several authors [4].

Figure 5(b) shows the distribution of $\hat{W}$ under the same condition as (a), but plotted in the vertical phase space $(V, v)$, where $(U, u)=\left(-x_{a} / \chi, 0\right)$ has been assumed. The number of linear ridges is half of those found in (a), because the contribution from the negative pole is negligible in this case.

Note that we have assumed $\sigma_{U}=18$ and $\sigma_{V}=1.8$ in the above discussions. This is to make the graphical plot more 
visible even in the wide range of interest, by means of broadening the individual ridges and eliminating the multilayer structure as discussed in Fig. 1(a).

\section{CORRELATION COEFFICIENT OF THE WIGNER FUNCTION}

The phase-space density discussed so far, which is defined by the Wigner function $\hat{W}$, is given as a function of the 4 coordinate variables: $U, V, u$, and $v$. For applications that require the distribution of the phase-space density, such as the ray-tracing simulation to design optical elements, $\hat{W}(U, V, u, v)$ is given as tabulated data computed at the 4D grid points, which may be probably stored in an external file. It is then imported to another code and is utilized to reproduce the distribution. Obviously, this process requires a lot of numerical cost. Assuming that we need $N_{p}$ points in one direction to accurately reproduce the distribution of $\hat{W}$, the total number of grid points, and thus the number of data points to be computed and stored in the external file, reaches $N_{p}^{4}$. If $\hat{W}$ can be decoupled to two functions representing the distributions in the horizontal and vertical phase spaces as in the case of $p$, i.e., the distribution function of the electron beam given in Eq. (14), the total data count reduces to $2 N_{p}^{2}$.

Let us introduce a distribution function $\hat{W}_{d}$ defined as

$$
\hat{W}_{d}(U, V, u, v) \equiv \frac{\hat{W}_{h}(U, u) \hat{W}_{v}(V, v)}{\hat{F}},
$$

with

$$
\begin{aligned}
& \hat{W}_{h}(U, u)=\iint \hat{W}(U, V, u, v) d V d v, \\
& \hat{W}_{v}(V, v)=\iint \hat{W}(U, V, u, v) d U d u,
\end{aligned}
$$

being the Wigner functions projected on the horizontal and vertical phase spaces, and

$$
\hat{F}=\iiint \int \hat{W}(U, V, u, v) d U d V d u d v,
$$

being the normalized total photon flux. Note that integration of $\hat{W}_{d}$ over the 4 D phase space equals that of $\hat{W}$, i.e., $\hat{F}$.

Integrating Eq. (17) over $V$ and $v$, it is easy to show that

$$
\begin{aligned}
\hat{W}_{h}(U, u)= & \frac{\sqrt{2 \pi}}{\sigma_{u}} \int \exp \left(-i u^{\prime \prime} U-\frac{\sigma_{U}^{2} u^{\prime \prime 2}}{2}\right) d u^{\prime \prime} \\
& \times \int \exp \left(-i \alpha_{u} u^{\prime} u^{\prime \prime}-\frac{u^{\prime 2}}{2 \sigma_{u}^{2}}\right) d u^{\prime} \\
& \times \int \hat{\mathcal{E}}^{*}\left(u-u^{\prime}+\frac{u^{\prime \prime}}{2}, v\right) \hat{\mathcal{E}}\left(u-u^{\prime}-\frac{u^{\prime \prime}}{2}, v\right) d v,
\end{aligned}
$$

and a similar expression for $\hat{W}_{h}(V, v)$. Note that the integration over $\eta$ has been omitted in the above equation for simplicity.

In order to examine if one can decouple the Wigner function and substitute $\hat{W}_{d}$ for $\hat{W}$, let us introduce a parameter $\kappa$ defined as follows

$$
\kappa=\frac{\left\langle\hat{W}_{d} \hat{W}\right\rangle}{\sqrt{\left\langle\hat{W}_{d}^{2}\right\rangle} \sqrt{\left\langle\hat{W}^{2}\right\rangle}},
$$

where $\langle f\rangle$ denotes the mean value of the function $f$ averaged over the range of interest in the phase space. This parameter, which can be regarded as the cross correlation coefficient in signal processing and statistics, ranges from -1 to 1 and gives us the information of how the two functions $\hat{W}_{d}$ and $\hat{W}$ are similar to each other. To be more specific, $\hat{W}$ can be well approximated by $\hat{W}_{d}$, if $\kappa$ is close to 1 .
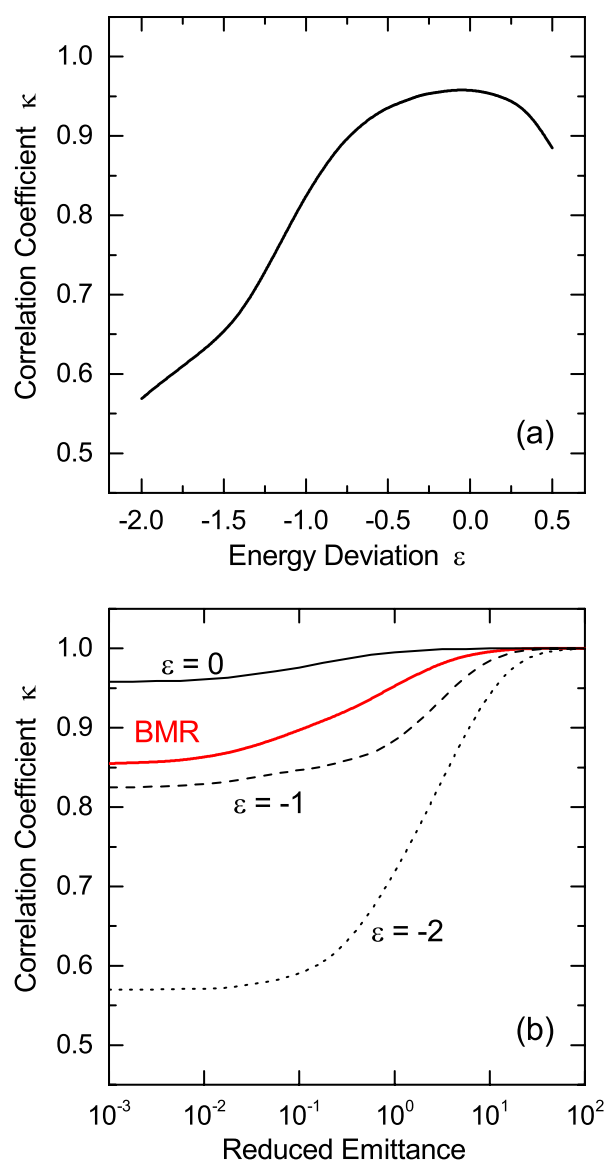

FIG. 6. Correlation coefficient $\kappa$ of the Wigner function in the case of UR, which is plotted as a function of (a) the energy deviation and (b) emittance of the electron beam for three different values of energy deviation. The red line in (b) shows that for BMR computed at the critical frequency. 
Figure 6 (a) shows the correlation coefficient $\kappa$ for UR emitted from a single electron, which has been computed as a function of the energy deviation parameter $\epsilon$. At the fundamental photon energy $(\epsilon=0), \kappa$ is relatively high $(\sim 0.96)$, while it rapidly drops for lower photon energies, meaning that approximation by $\hat{W}_{d}$ is less accurate.

It should be noted that the above discussion should be more or less modified when we take into account the effects due to the finite emittance of the electron beam, whose distribution function is supposed to be decoupled. In order to investigate such an effect, the correlation coefficients at $\epsilon=0,-1$, and -2 have been computed as a function of the electron emittance. For simplicity, we set up the related parameters as follows: $\hat{\alpha}_{u, v}=0, \sigma_{U, V} / \sigma_{u, v}=10$, and $\hat{\varepsilon}_{v} / \hat{\varepsilon}_{h}=0.1$, where $\hat{\varepsilon}_{h, v}$ is defined as

$$
\hat{\varepsilon}_{h, v} \equiv 2 \sigma_{U, V} \sigma_{u, v},
$$

which denotes the electron beam emittance normalized by the emittance of diffraction-limited light $(\lambda / 4 \pi)$, and is referred to as the reduced emittance. The results of computation are shown in Fig. 6(b) as solid, dashed, and dotted black lines. We find that $\kappa$ rapidly approaches one when $\hat{\varepsilon}_{h}$ becomes larger than unity. This result also applies to BMR, as indicated by the red line in the same figure.

Note that the above discussion is valid only for the conditions under consideration and may not be universal. Even so, we may conclude that if the electron beam emittance is relatively larger than the optical emittance at the target wavelength, the Wigner function can be decoupled and $\hat{W}_{k}$ can be utilized instead of $\hat{W}$.

\section{EXAMPLE: OPTIMIZING THE BETATRON FUNCTIONS}

As a practical application of the numerical method described so far, let us consider the process to optimize the betatron functions and maximize the brilliance of UR. Table I summarizes the electron and undulator parameters

TABLE I. Electron beam and undulator parameters used in the computation to optimize the betatron functions.

\begin{tabular}{lc}
\hline \hline Parameter & Value \\
\hline Electron beam & \\
Energy & $6 \mathrm{GeV}$ \\
Natural emittance & $10^{-10} \mathrm{~m} \cdot \mathrm{rad}$ \\
Coupling constant & 0.1 \\
Energy spread & $10^{-3}$ \\
Betatron functions & $1 \mathrm{~m}$ \\
Undulator & \\
Period & $20 \mathrm{~mm}$ \\
$K$ value & 1.19 \\
Length & $4 \mathrm{~m}$ \\
Fundamental energy & $10 \mathrm{keV}$ \\
\hline \hline
\end{tabular}

assumed in the computation, which are typical design values in SR facilities currently under planning.

Before discussions on the result of optimization based on the Wigner function method, let us first recall the traditional method of optimization based on the Gaussian approximation. It is well known that the betatron function of the electron beam is optimized when it equals that of the photon beam emitted from a single electron. This gives the optimum betatron function $\beta_{\mathrm{opt}, G}$ based on the Gaussian approximation, which is defined as

$$
\beta_{\mathrm{opt}, G}=\frac{\sigma_{n r}}{\sigma_{n r^{\prime}}}=\frac{L}{2 \pi} .
$$

For example, we have $\beta_{\mathrm{opt}, G}=0.64 \mathrm{~m}$ when the undulator is $4 \mathrm{~m}$ long. Note that this discussion is validated only at the $n$th harmonic photon energies because the angular divergence and source size of UR are not clearly defined at other energies, as already mentioned in Sec. III B. To be more specific, $\beta_{\mathrm{opt}, G}$ at these detuned photon energies cannot be defined by the traditional method.

Now let us turn to the optimization based on the Wigner function method. Because the electron emittance is about one order of magnitude larger than the optical emittance, it is reasonable to decouple the Wigner function as discussed in Sec. IV and thus we look for the betatron functions that maximize the projected Wigner functions $\hat{W}_{h, v}$.

Figures 7(a) and (b) show the on-axis Wigner function, $\hat{W}_{h, v}(0,0)$, computed at the fundamental photon energy $\left(\hbar \omega_{0}=10 \mathrm{keV}\right)$ as a function of $\beta_{x, y}$. The dashed lines indicate $\beta_{x, y}=0.64 \mathrm{~m}$, being the optimum condition determined by the Gaussian approximation. We find that the actual optimum value is slightly shifted to larger value $\left(\beta_{x, y} \sim 1 \mathrm{~m}\right)$.

In the above optimization, the photon energy has been fixed at the fundamental energy of UR in order to compare with the traditional method. In practice, we can tune the photon energy to maximize the brilliance as well as the betatron functions. Figures 7 (c) and (d) show the contour plot of $\hat{W}_{h, v}(0,0)$ as a function of $\beta_{x, y}$ and $\hbar \omega$, where we find that it is maximized when the photon energy is slightly detuned to lower energy ( $\hbar \omega \sim 9.97 \mathrm{keV}$ ). This is consistent with the fact that the total photon flux of UR is nearly doubled when the photon energy is detuned from $\hbar \omega_{0}$ to some lower optimum energy [4].

Note that the optimum betatron function at this optimum photon energy $\left(\hbar \omega_{\text {opt }}\right)$ is shifted from $1 \mathrm{~m}$ to $0.7 \mathrm{~m}$. This means that the optimum betatron function at the photon energy of $\hbar \omega_{\text {opt }}$, which is determined by the Wigner function method, is close to $\beta_{\mathrm{opt}, G}$, i.e., the optimum value roughly determined by the Gaussian approximation without taking into account the energy detuning. Although this result sounds useful and convenient, we have to take care that it has not been proven to be universal. 


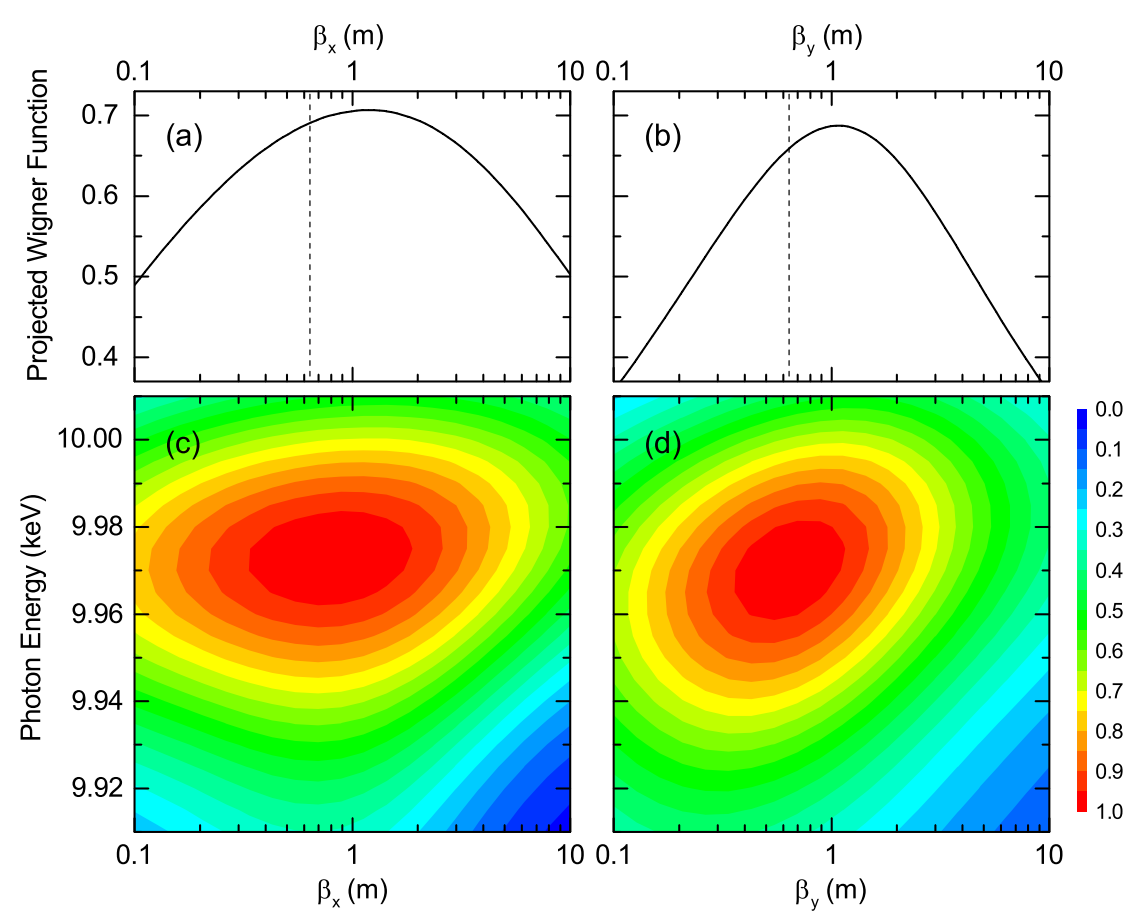

FIG. 7. Optimization of betatron functions. The projected Wigner functions $\hat{W}_{h, v}(0,0)$ are computed as a function of the betatron functions and photon energy. The results at the fixed photon energy of $10 \mathrm{keV}$ are plotted in (a) and (b), while the contour plots are shown in (c) and (d).

\section{SUMMARY}

We have described the numerical methods to compute the phase-space density based on the Wigner function method. In addition to the optimization of betatron functions discussed in Sec. V, there exist a large number of practical applications, such as an exact evaluation of brilliance and its comparison between facilities, an accurate computation of the photon distribution at the source point to be utilized in the external ray-tracing program, and so on.

In this paper, the Wigner function at the source point $W(\boldsymbol{\rho}, \boldsymbol{\theta}, z=0)$ has been described. It is worth noting that once it is given, the Wigner function at any longitudinal position $z=Z$ is easily evaluated by using the relation $W(\boldsymbol{\rho}, \boldsymbol{\theta}, z=Z)=W(\boldsymbol{\rho}-Z \boldsymbol{\theta}, \boldsymbol{\theta}, z=0)$. Also note that the cross-spectral density can be computed by Fouriertransforming the Wigner function, which is useful to describe the propagation of radiation based on the wave optics.

It should be mentioned that the Wigner function is not positive definite. This can be problematic for the ray-trace simulation because the meaning of the negative phasespace density is not clear. One solution is to transfer all the photons to the target position based on the geometrical optics, then sum up them with an adequate weight reflecting the phase-space density, which is not necessarily positive. Another solution is to compute the cross-spectral density and transfer it according to wave optics.

Finally we note that all the methods presented in this paper have been implemented in the SR calculation code
SPECTRA [15], which has been developed and maintained in SPring-8, and is freely available.

\section{APPENDIX: ELECTRON DISTRIBUTION FUNCTION EXPRESSED IN THE NORMALIZED COORDINATES}

The distribution function of the electron beam at the nonzero dispersion section is given here in terms of the normalized coordinates.

The distribution function of the Gaussian beam in the horizontal phase space $\left(x, \theta_{x}\right)$ without energy spread is in general given as

$$
F_{0}\left(x, \theta_{x}\right)=\frac{1}{2 \pi \varepsilon_{x}} \exp \left(-\frac{\gamma_{x} x^{2}+2 \alpha_{x} x \theta_{x}+\beta_{x} \theta_{x}^{2}}{2 \varepsilon_{x}}\right),
$$

where $\alpha_{x}, \beta_{x}$, and $\gamma_{x}$ are the so-called Twiss parameters, and $\varepsilon_{x}$ denotes the beam emittance. In order to take into account the finite energy spread, the above function should be convoluted with

$$
E\left(x, \theta_{x}\right)=\frac{1}{2 \pi \sigma_{e} \sigma_{e^{\prime}}} \exp \left(-\frac{x^{2}}{2 \sigma_{e}^{2}}-\frac{\theta_{x}^{2}}{2 \sigma_{e^{\prime}}^{2}}\right),
$$

with

$$
\sigma_{e}=\eta_{x} \sigma_{\gamma}, \sigma_{e^{\prime}}=\eta_{x}^{\prime} \sigma_{\gamma},
$$

where $\eta_{x}$ and $\eta_{x}{ }^{\prime}$ are the dispersion functions, and $\sigma_{\gamma}$ denotes the energy spread of the electron beam. The 
convolution can be done analytically to give the distribution function

$$
\begin{aligned}
& F\left(x, \theta_{x}\right) \\
& =\frac{1}{2 \pi \Gamma} \exp \left[-\frac{\left(\gamma_{x} \varepsilon_{x}+\sigma_{e^{\prime}}^{2}\right) x^{2}+2 \alpha_{x} \varepsilon_{x} x \theta_{x}+\left(\beta_{x} \varepsilon_{x}+\sigma_{e}^{2}\right) \theta_{x}^{2}}{2 \Gamma^{2}}\right],
\end{aligned}
$$

with

$$
\Gamma^{2}=\left(\varepsilon_{x} \gamma_{x}^{-1}+\sigma_{e}^{2}\right)\left(\varepsilon_{x} \gamma_{x}+\sigma_{e^{\prime}}^{2}\right) .
$$

Introducing the normalization factors $\chi$ and $\chi^{\prime}$, the distribution function can be modified to Eq. (15), with parameters given as follows

$$
\begin{gathered}
\sigma_{U}=\frac{\sqrt{\varepsilon_{x} \gamma_{x}^{-1}+\sigma_{e}^{2}}}{\chi}, \\
\sigma_{u}=\frac{\sqrt{\varepsilon_{x} \gamma_{x}+\sigma_{e^{\prime}}^{2}}}{\chi^{\prime}}, \\
\hat{\alpha_{u}}=\frac{\alpha_{x} \varepsilon_{x}}{\varepsilon_{x} \gamma_{x}+\sigma_{e^{\prime}}^{2}} \frac{\chi^{\prime}}{\chi} .
\end{gathered}
$$

Note that derivation of another Eq. (16) for the vertical direction and definitions of the related parameters are straightforward.
[1] G. Geloni, E. Saldin, E. Schneidmiller and M. Yurkov, Nucl. Instrum. Methods Phys. Res., Sect. A 588, 463 (2008).

[2] I. A. Vartanyants and A. Singer, New J. Phys. 12, 035004 (2010).

[3] W. Wigner, Phys. Rev. 40, 749 (1932).

[4] R. Coisson and R. P. Walker, Proc. SPIE Int. Soc. Opt. Eng. 582, 24 (1985).

[5] K. J. Kim, Nucl. Instrum. Methods Phys. Res., Sect. A 246, 71 (1986).

[6] K. J. Kim, AIP Conf. Proc. 184, 565 (1989).

[7] J. Bahrdt, Appl. Opt. 36, 4367 (1997).

[8] T. Tanaka and H. Kitamura, AIP Conf. Proc. 879, 355 (2007).

[9] I. V. Bazarov, Phys. Rev. ST Accel. Beams 15, 050703 (2012).

[10] A. Gasbarro and I. V. Bazarov, J. Synchrotron Radiat. 21, 289 (2014).

[11] O. V. Chubar, Rev. Sci. Instrum. 66, 1872 (1995).

[12] See for example, M. Born and E. Wolf, Principles of Optics (Pergamon Press, New York, 1984).

[13] See for example, A. Yariv, Optical Electronics in Modern Communications (Oxford University Press, New York, 1997).

[14] J. Schwinger, Phys. Rev. 75, 1912 (1949).

[15] T. Tanaka and H. Kitamura, J. Synchrotron Radiat. 8, 1221 (2001); SPECTRA is freely available from http://radiant .harima.riken.go.jp/spectra/index.html. 